\title{
AKT2 Gene Polymorphisms, Srankl/OPG And Hormone Measurements in Polycystic Ovarian Syndrome (PCOS) Women
}

Aikaterini Zerva ${ }^{1}$, Christos Kroupis ${ }^{1^{*}}$, Efthihios Trakakis ${ }^{2}$, Nikoleta Poumpouridou ${ }^{1}$, Marina Tsagkla ${ }^{1}$, Evanthia Kassi ${ }^{3}$, Dimitrios Kassanos ${ }^{4}$ and Kleanth Dima $^{1}$

${ }^{1}$ Department of Clinical Biochemistry, Attikon University General Hospital, Medical School, National and Kapodistrian University of Athens, Athens, Greece

${ }^{2}$ Department of Obstetrics and Gynecology, Attikon University General Hospital, Medical School, National and Kapodistrian University of Athens, Athens, Greece

${ }^{3}$ Department of Biological Chemistry, Medical School, National and Kapodistrian University of Athens, Athens, Greece

${ }^{4}$ Fetal Medicine Unit, Third Department of Obstetrics and Gynecology, Attikon University General Hospital, Medical School, National and Kapodistrian University of Athens, Athens, Greece.

*Corresponding author: Christos Kroupis, Department of Clinical Biochemistry, Attikon University General Hospital, Medical School, National and Kapodistrian University of Athens, Athens, Greece, Tel: ; E-mail: ckroupis@med.uoa.gr

Received date: February 06, 2017; Accepted date: Feb 24, 2017; Published date: March 01, 2017

Copyright: (c) 2017 Zerva A, et al. This is an open-access article distributed under the terms of the Creative Commons Attribution License, which permits unrestricted use, distribution, and reproduction in any medium, provided the original author and source are credited.

\section{Abstract}

Objective: Polycystic Ovarian Syndrome (PCOS) is a common endocrinologic disorder diagnosed in $6-10 \%$ of female population at reproductive age. Altered expression of $A K T 2$ gene has been correlated to increased insulin tolerance and reduced glucose disposal, both of which are PCOS features. The goal of this study was to investigate the association between AKT2 gene polymorphisms, serum biomarkers OPG and SRANKL as cardiovascular biomarkers, hormones (DHEAS, SHBG, testosterone, E2, LH, FSH, prolactin, insulin, 17-OH progesterone) and clinical characteristics as well (amenorrhea, oligomenorrhea, dysmenorrhea, acne, hirsutism, oily skin).

Study Design: A total of 60 Greek Caucasian PCOS patients and another 30 healthy women that were age- and BMI-matched, were recruited in the study and their blood specimens and clinical characteristics collected. Serum OPG and SRANKL were measured with ELISA kits and hormones with Roche Cobas e411 immunochemical analyzer. Four AKT2 gene DNA SNPs (Single Nucleotide polymorphisms) were selected; rs11671439, rs8100018, rs3730051 and rs2304188 and novel real-time qPCR methods were developed using either the dual probe or the single probe format for their genotyping in the LightCycler platform. All findings were confirmed with DNA Sequencing and statistical analysis was performed using SNPstats and SPSS software.

Results: PCOS women had higher serum levels of SRANKL, DHEAS, testosterone and 17-OH progesterone and lower levels of E2, SHBG and prolactin than controls. There was a statistically important difference for SNP rs2304188 between patients and controls regarding Minor Allele Frequency (MAF\%): it was found to be more frequent in PCOS patients compared to controls [OR 4.04 (C.I. 1.12-14.54)], whereas concerning the entire population, studied individuals bearing the rs2304188 SNP have higher values of DHEAS and 17-OH progesterone, both biomarkers of PCOS. Furthermore, an association between hirsutism and SNP rs2304188 was found in PCOS patients $(p=0.044)$. Eight participants had SNP rs8100018 in their DNA in combination with either rs11671439 or rs2304188; all were PCOS women.

Conclusions: Our results adjudicate further study on AKT2 gene SNPs in relation with PCOS

Keywords: Polycystic ovary syndrome (PCOS); AKT2 gene; SNP; sRANKL; OPG

\section{Abbreviations \\ BMI: Body Mass Index; CI: confidence intervals; HWE: Hardy Weinberg Equilibrium; GVS: Genome Variation Server: MAF: Minor Allele Frequency; OPG: osteoprotegerin; OR: Odds ratio; PCOS: Polycystic Ovarian Syndrome; PCR: Polymerase Chain Reaction; POM: Polycystic ovarian morphology; qPCR: quantitative Polymerase Chain Reaction; sRANKL: soluble receptor activator of nuclear factor (NF)-kB ligand; SNP: Single Nucleotide Polymorphism; SD: Standard Deviation; Tm: Melting Point.}

\section{Introduction}

Polycystic ovarian syndrome (PCOS) is a common endocrinologic disorder diagnosed in $6-10 \%$ of the female population at reproductive age [1-2]. Although many studies have investigated PCOS prevalence, there are discrepancies in their results, partly due to the use of various definitions of the syndrome and its sub-phenotypes, differences between study cohorts, ethnicities, and types of recruitment and sampling. PCOS is characterized by hyperandrogenism, oligoovulation, and polycystic ovarian morphology [3-4].

In 1990 the National Institute of Health (NIH) proposed the first PCOS diagnostic criteria that required the combination of chronic oligo/anovulation and clinical or biochemical evidence of hyperandrogenism, with the exclusion of related disease such as nonclassical congenital adrenal hypertrophy, Cushing syndrome, 
hyperprolactinemia and malfunctions of the thyroid gland [5] Subsequently, in 2003 the Rotterdam European Society of Human Reproduction and the Embryology/American Society for Reproduction Medicine (ESHRE/ASRM) sponsored the PCOS Consensus Workshop group (Rott-PCOS) that proposed the addition of polycystic ovarian morphology (POM) to the NIH criteria, stating that PCOS could be diagnosed when any two of these three criteria were present [6]. More recently, in 2006 the Androgen Excess Society (AES) proposed new diagnostic criteria and stated that the syndrome must only be diagnosed in the presence of hyperanderogenism in combination with oligo/anovulation and/or POM [7]. Although polycystic ovarian syndrome is probably one of the most common endocrinologic disorder, its pathogenesis still remains unclear.

Obesity, insulin resistance and low vitamin D levels are present in more than $50 \%$ of PCOS patients. These factors along with hyperandrogonism could have adverse effects in their long-term health.

Insulin resistance is observed in up to $70 \%$ of women with PCOS. The molecular basis of this intrinsic resistance mechanism remains unknown, but post-insulin receptor defects appear to contribute to the pathophysiology of both insulin resistance and hyperandrogonemia [8]. The insulin signaling pathway includes two main arms: a metabolic arm through the activation of phosphatidylinositol 3-kinase (PI3K) and a mitogenic arm acting via mitogen-activated protein kinase [9]. The expression and activity of PI3K downstream targets have been investigated in PCOS tissues, with distinct molecular defects in post-insulin receptor signaling identified in fibroblasts and adipose tissues of PCOS women [10]. AKT gene activates 3-phosphoinositide dependent kinase (PIDK), which is activated by the product of PI3K, phosphatidylinositol 3,4,5-trisphosphate and participates in insulin signaling. Three highly conserved genes encode the forms of $A K T 1$, $A K T 2$ and $A K T 3$ [7,11]. AKT2 (AKT serine/threonine kinase 2) is located at 19q13.2 locus (22 exons) and is a widely expressed gene that is involved in metabolism of insulin, mitogenic signaling and apoptosis [11]. In type 2 diabetes, there is reduced activation of AKT2 gene by insulin in adipocytes. Insulin-stimulated AKT phosphorylation is reduced $40-60 \%$ in PCOS skeletal muscle compared with matched control muscle [9].

It has traditionally been suggested that prolactin excess, enzymatic steroidogenic abnormalities and thyroid disorders need to be excluded before a PCOS diagnosis is confirmed. However, there is paucity of data regarding the prevalence of PCOS phenotype in some of these disorders, whereas other endocrine diseases that exhibit PCOS-like features may evade diagnosis and proper management, if not considered. Hyperinflammation and impaired epithelial function were reported to a larger extent in PCOS women and could be particularly associated with hyperandrogenism [12], obesity and insulin resistance. Available data from register-based and data linkage studies support that metabolic-vascular and thyroid diseases, asthma, migraine, depression, and cancer are diagnosed more frequently in PCOS, whereas fracture risk is decreased [13]. Drug prescriptions are significantly more common in PCOS than controls within all diagnostic categories including antibiotics use. The causal relationship between PCOS and autoimmune disease represents an interesting new area of research. PCOS is a lifelong condition and long-term morbidity that could be worsened by obesity, sedentary lifestyle, western-type diet and smoking, whereas lifestyle intervention including weight loss may partly or fully resolve the PCOS symptoms and could improve the long-term prognosis. As many as $20 \%$ of women with infertility problems (including fecundability and early pregnancy loss) have been diagnosed with PCOS. It is often called the most common cause of anovulatory infertility in women.

PCOS is also associated with cardiovascular problems, neurological and psychological effects on quality of life (including anxiety and depression), and breast and endometrial cancers [14]. Recently there was a connection between cardiovascular disease and osteoprotegerin (OPG, an osteoclast inhibitory factor) and sRANKL (soluble receptor activator of nuclear factor (NF)-kB ligand) biomarkers [15]. There is no known PCOS cause; however, there has been evidence that shows that both environmental as well as genetic factors play a role in the etiology [16].

The goal of this study was to investigate the association among AKT2 gene SNPs (Single Nucleotide Polymorphisms), OPG/sRANKL as cardiovascular biomarkers, hormone measurements and clinical characteristics as well in PCOS patients in the Greek female population.

\section{Materials and Methods}

\section{Subjects}

We studied a total of 60 Caucasian patients with PCOS aged 18-35 and 30 Caucasian control women that were age-and BMI-matched. Weight and height were measured and obesity was assessed by estimating Body Mass Index (BMI). All patients were recruited from the reproductive endocrinology clinic of Attikon University General Hospital (physician in charge: E.T.). Participation in the research protocol was offered to patients meeting the PCOS Rotterdam diagnostic criteria and the following inclusion criteria: being premenopausal, not pregnant and not submitted to hormonal therapy (including oral contraceptives) for at least 3 months prior to testing. Control subjects were healthy women working at Attikon University General Hospital with regular menstrual cycles and without any family history of PCOS or hirsutism. They had no evidence of hirsutism, acne, alopecia, endocrine dysfunction and were not submitted to hormonal therapy (including oral contraceptives) for at least 3 months prior to testing. A standard questionnaire was filled, including menstural periods and irregularity, hirsutism and acne, reproductive history, gynecological history and use of medications including oral contraceptive pills. The presence of hirsutism was scored in all subjects using the Lorenzo Scale [17]. Subjects with diabetes were excluded. Transvaginal ultrasonography was performed in all subjects. The ethics committee of the hospital approved the study and a signed informed consent was obtained from each participant.

\section{Methods}

\section{Serum measurements}

Whole blood was obtained on day 2 or 3 of the menstrual cycle or during amenorrhea, centrifuged for $10 \mathrm{~min}$ at $3500 \mathrm{rpm}$ and serum was collected and stored in $-80^{\circ} \mathrm{C}$ until assayed.

Hormone measurements included DHEAS, SHBG, testosterone, E2, LH, FSH, T3, T4, TSH, FT3, FT4, insulin, prolactin and were performed on an automated routine immunochemical analyzer COBAS e-411 (Roche Diagnostics, Manheim, Germany) that employs electrochemiluminescence. 
Serum OPG and sRANKL were measured by commercial ELISA kits (Biomedica Gruppe, Vienna, Austria). The limit of detection for OPG was $0.14 \mathrm{pmol} / \mathrm{L}$ and that of sRANKL $0.02 \mathrm{pmol} / \mathrm{L}$. Both kits show inter-assay precisions of less than $8 \%$ in their performance characteristics.

\section{Genomic DNA isolation}

From an additional blood sample collected in EDTA tubes, genomic DNA was isolated from $200 \mu \mathrm{L}$ by the QIAamp DNA Blood Mini kit (Qiagen, Germany) according to the manufacturer's instructions. DNA purity and quantity were determined by fluorescence readings with the use of Quant-iTdsDNA-BR kit in the Qubit 1.0 fluorimeter (Life
Technologies Invitrogen, USA). Extracted DNA was stored in $-20^{\circ} \mathrm{C}$ until assayed.

\section{Genotyping}

Four intronic SNPs (rs2304188, rs11671439, rs3730051 and rs8100018) spanning the entire 51.5-kb genomic length of $A K T 2$ gene were selected for evaluation of their association with the disease (Table 1). We developed novel real-time qPCR methods for their genotyping by using either the dual or single hybridization probe format. The sequences of the primers and probes of the proposed real time PCR assays synthesized by TIB MOLBIOL (Berlin, Germany) are shown in Table 1.

\begin{tabular}{|c|c|c|c|c|}
\hline SNP & Location & PCR primers & Probes & Tanneal \\
\hline \multirow[t]{2}{*}{ rs2304188 } & Intron 10 & GTGGGGGTAGGAACGTG & CCCCAGACCTGTGTGT X̌İ TTCCA & 56 \\
\hline & & GAGGCTGGCATCACAGTC & & \\
\hline \multirow[t]{2}{*}{ rs11671439 } & Intron 1 & CCTGCATTAGCCTGTGATGAA & GGGTCCTTGECTTGCCCAC-FL & 59 \\
\hline & & СССTATGACTTCATCTCAGGAAGTAC & LC 640-TTCTAGACTCCCAAGCCTCCGTTTCCT & \\
\hline \multirow[t]{2}{*}{ rs8100018 } & Intron 4 & GAAAAAAGCCTGTCATGGA & AAAAAA XI×AGCAGGGGCCGGGGAC & 53 \\
\hline & & AACTTGCTTTAACCCATGC & & \\
\hline \multirow[t]{2}{*}{ rs3730051 } & Intron 8 & TTGTGATGGAGTATGCCAACG & ACGAAGCCGCCTGCCTCAAGGG-FL & 59 \\
\hline & & TGGGCAAGCCACTTAACCT & LC705-GAGGGGCACCAGATGACACTGA & \\
\hline
\end{tabular}

Table 1: Primers and probe sequences used in $A K T 2$ genotyping analysis. The SNP position is underlined. Probes were labeled either with fluorescein (FL) or with LC 640 or 705 dyes. ${ }^{*}$ XI denotes FL.

Real-time PCR was performed in glass capillary tubes in the LightCycler 1.5 instrument (Roche Applied Science, Switzerland). The amplification mixture of a total volume of $10 \mu \mathrm{L}$ included $1 \mu \mathrm{L}$ of genomic DNA extract as template $(\sim 30 \mathrm{ng}), 2 \mathrm{pmol} / \mu \mathrm{L}$ forward primer, $2 \mathrm{pmol} / \mu \mathrm{L}$ reverse primer, either $0.1 \mu \mathrm{M}$ Simple probe or a combination of $0.15 \mu \mathrm{M}$ FL-Sensor probe with fluorescein and $0.15 \mu \mathrm{M}$ Anchor probe labeled with LC640 or LC705 dye (in the case of dual probe format) and $1 \mu \mathrm{L}$ of $10 \times$ FastStart DNA Master Hybprobe mix (Roche Applied Science, Switzerland). We added $\mathrm{MgCl} 2$ to a final optimal 3 $\mathrm{mM}$ concentration. Sterile $\mathrm{H}_{2} \mathrm{O}$ was used to supplement up to $10 \mu \mathrm{L}$. The cycling protocol consists of pre-incubation at $95^{\circ} \mathrm{C}$ for $10 \mathrm{~min}$ for hot-start activation, followed by 40 cycles of denaturation at $95^{\circ} \mathrm{C}$ for 10 secs, annealing at a specified temperature (Table 1 for Tanneal) for $20 \mathrm{sec}$ and extension at $72^{\circ} \mathrm{C}$ at $30 \mathrm{sec}$. Emitted fluorescence was measured at the end of each annealing step at a selected fluorescence channel (F1 for simple probes, F2 for LC640 or F3 for LC705 anchor probe). Immediately after amplification, melting curve analysis was performed on the LightCycler. The melting curve protocol included raising the temperature at $95^{\circ} \mathrm{C}$ for 10 secs, cooling at $40^{\circ} \mathrm{C}$ for $120 \mathrm{sec}$ and slow heating to $85^{\circ} \mathrm{C}$ at a rate of $0.3^{\circ} \mathrm{C} / \mathrm{sec}$, during which time fluorescence measurements were continuously collected in the selected fluorescence channel. The first derivative plot $(-\mathrm{d}(\mathrm{F}) / \mathrm{dT}$ vs. $\mathrm{T})$ is then used for easy identification of wild-type and mutant alleles by their different peaks, as the mismatched probe will dehybridize at an earlier stage.

\section{DNA Sequencing}

For the verification of the real-time qPCR results, the Gold Standard method of DNA Sequencing was used for method comparison. After the purification of the amplicons prepared from conventional PCR reactions (High Pure PCR Cleanup Micro kit, Roche Applied Science, Switzerland), cycle sequencing reaction were performed with the Big Dye 1.1 reagent in both directions with the use of either the forward or the reverse primer (ThermoFisher Applied Biosystems, USA). $10 \mu \mathrm{L}$ of the purified cycle sequencing reactions (by NucleoSeq columns, Macherey-Nagel, Germany) were heated at 95 for $2 \mathrm{~min}$ and cooled immediately at 4 for $2 \mathrm{~min}$ with $10 \mu \mathrm{L}$ formamide and then run in capillaries of the ABI Prism 310 Genetic Analyzer. For the analysis of DNA sequencing electropherograms the Chromas 2.01 software was used (Technelysium Pty Ltd, Australia) and results were compared with the expected gene sequences with the NCBI BLAST tool (https:// blast.ncbi.nlm.nih.gov).

\section{Statistics}

Genotyping statistical analysis was performed through the SNPStats Internet platform (http://bioinfo.iconcologia.net/snpstats/start.html). The variants were tested for Hardy-Weinberg equilibrium (HWE) in either POCS patients or controls for each studied SNP, tables with genotype and allele frequencies were constructed and Odds Ratios (OR) and 95\% confidence intervals (CI) were calculated.

Further analysis was performed with SPSS v.22 (IBM, USA). Descriptives were calculated for all variables. The distribution of continuous variables was assessed with Kolmogorov-Smirnov test. 
Citation: Zerva A, Kroupis C, Trakakis E, Poumpouridou N, Tsagkla M, et al. (2017) AKT2 Gene Polymorphisms, Srankl/OPG And Hormone Measurements in Polycystic Ovarian Syndrome (PCOS) Women. Clin Med Biochem 3: 123. doi:10.4172/2471-2663.1000123

Page 4 of 7

Comparisons between continuous variables non-normally distributed were performed with Mann-Whitney U. Dichotomous comparisons were performed using Fisher's exact test. The level of significance was set at $\mathrm{p} \leq 0.05$.

\section{Results}

Cases and controls were matched for age and BMI (Table 2, $\mathrm{p}>0.05$, Mann Whitney). Almost all PCOS clinical characteristics except amenorrhea were significantly more frequent for cases than controls as calculated with Fisher's exact test (Table 2). Cases had significantly higher values for sRANKL $(\mathrm{p}<0.000)$, DHEAS $(\mathrm{p}<0.000), 17-\mathrm{OH}-$ progesterone $(\mathrm{p}<0.000)$, and testosterone $(\mathrm{p}=0.021)$, and significantly lower values for SHBG $(\mathrm{p}=0.003), \mathrm{E} 2(\mathrm{p}<0.000)$ and PRL $(\mathrm{p}=0.012)$ in comparison to controls (Table 3 ).

\begin{tabular}{|l|l|l|l|}
\hline & Cases & Controls & $\mathbf{p}$ \\
\hline & Mean (SD) & Mean (SD) & \\
\hline Age & $28.18(3.53)$ & $29.07(3.53)$ & 0.289 \\
\hline BMI & $24.63(4.76)$ & $24.71(3.81)$ & 0.585 \\
\hline & $\%$ & $\%$ & \\
\hline Amenorrhea & 15 & 3.33 & 0.121 \\
\hline Oligomenorrhoea & 40 & 3.33 & $<0.000$ \\
\hline Dysmenorrhea & 60 & 0 & $<0.000$ \\
\hline Acne & 60 & 0 & $<0.000$ \\
\hline Hirsutism & 63.3 & 0 & $<0.000$ \\
\hline Oily skin & 40 & 6.66 & $<0.000$ \\
\hline
\end{tabular}

Table 2: Differences in clinical characteristics between PCOS cases and controls.

All novel $A K T 2$ gene genotyping methods were optimized and developed satisfactorily and were validated for their accuracy with the Gold Standard DNA sequencing (100\% concordance). Characteristic figures with amplification plot, melting curves with Tm (melting point) allele determination and DNA Sequencing electrophore grams e.g., for SNP rs3730051 are shown in Supplementary Figures 1,2,3 respectively. Each real-time PCR reaction included a blank and either a homozygote or a heterozygote positive control.

\begin{tabular}{|l|c|c|c|c|l|}
\hline & \multicolumn{2}{l|}{ Controls } & \multicolumn{2}{l|}{ Cases } & $p$ \\
\hline & \multicolumn{2}{|l|}{ Mean (SD) } & \multicolumn{2}{l|}{ Mean (SD) } & \\
\hline sRANKL & 0.01 & $(0.01)$ & 0.47 & $(0.72)$ & $p<0.000^{*}$ \\
\hline OPG & 2.99 & $(0.75)$ & 2.76 & $(1.47)$ & $p=0.205$ \\
\hline DHEAS & 212.83 & $(42.59)$ & 325.20 & $(132.66)$ & $p<0.000^{*}$ \\
\hline SHBG & 66.63 & $(14.95)$ & 55.90 & $(34.43)$ & $\mathrm{p}=0.003^{*}$ \\
\hline Testosterone & 0.65 & $(1.23)$ & 3.07 & $(4.31)$ & $\mathrm{p}=0.021^{*}$ \\
\hline E2 & 58.79 & $(9.56)$ & 46.20 & $(15.38)$ & $\mathrm{p}<0.000^{*}$ \\
\hline LH & 6.24 & $(0.87)$ & 6.97 & $(4.25)$ & $\mathrm{p}=0.583$ \\
\hline FSH & 5.26 & $(0.45)$ & 5.71 & $(1.31)$ & $\mathrm{p}=0.127$ \\
\hline Prolactin & 14.42 & $(0.68)$ & 17.31 & $(7.11)$ & $\mathrm{p}=0.012^{*}$ \\
\hline Insulin & 8.62 & $(2.04)$ & 10.66 & $(7.91)$ & $\mathrm{p}=0.728$ \\
\hline 17-OH Progesterone & 0.30 & $(0.05)$ & 0.96 & $(0.50)$ & $\mathrm{p}<0.000^{*}$ \\
\hline
\end{tabular}

Table 3: Differences in serum biochemical parameters between PCOS cases and controls. ${ }^{*}$ denotes statistical significance.

All SNP genotyping results were in HWE ( $\mathrm{p}>0.05)$. Minor Allele frequencies (MAF\%) were separately calculated for cases and controls and also compared to those given by the Genome Variation Server (GVS) from data collected by analyzing a minimum of 450 genomes per SNP. Only one of the SNPs showed statistical significant difference with PCOS: rs2304188 T minor allele increased from 9\% in controls to $29 \%$ in patients providing an OR 4.04 (C.I. 1.12-14.54) for disease risk to the $\mathrm{T}$ allele carrier versus the wild-type carrier (Figure 1 and Table 4). Differences in MAF\% between the GVS and the current study were significant for rs2304188 (controls only) and rs3730051 (both cases and controls).

\begin{tabular}{|l|l|l|l|l|l|l|l|l|}
\hline SNP & Alleles & GVS MAF $\%$ & $\begin{array}{l}\text { Control MAF } \\
\%\end{array}$ & Case MAF $\%$ & $\mathbf{p}^{*}$ & $\mathbf{p}^{* *}$ & $\mathbf{p}^{* * *}$ & OR (C.I.) \\
\hline rs2304188 & C/T & 24 & 9.4 & 26.7 & 0.014 & 0.870 & 0.016 & $4.04(1.12-14.54)$ \\
\hline rs8100018 & C/G & 29 & 37.5 & 41.7 & 0.231 & 0.076 & 0.540 & $1.29(0.56-2.99)$ \\
\hline rs11671439 & C/T & 18 & 18.8 & 23.3 & 1.000 & 0.484 & 0.480 & $1.37(0.55-3.42)$ \\
\hline rs3730051 & A/G & 21 & 37.5 & 41.7 & 0.013 & 0.002 & 0.970 & $0.99(0.50-1.95)$ \\
\hline
\end{tabular}

Table 4: AKT2 gene SNPs descriptive statistics for PCOS case and control participants versus GVS data. *Fisher's exact test between the study controls' MAF\% and GVS MAF\%. ${ }^{* *}$ Fisher's exact test between the study cases' MAF\% and GVS MAF\%. ${ }^{* *}$ Fisher's exact test between the study cases' MAF\% and controls' MAF\%. 


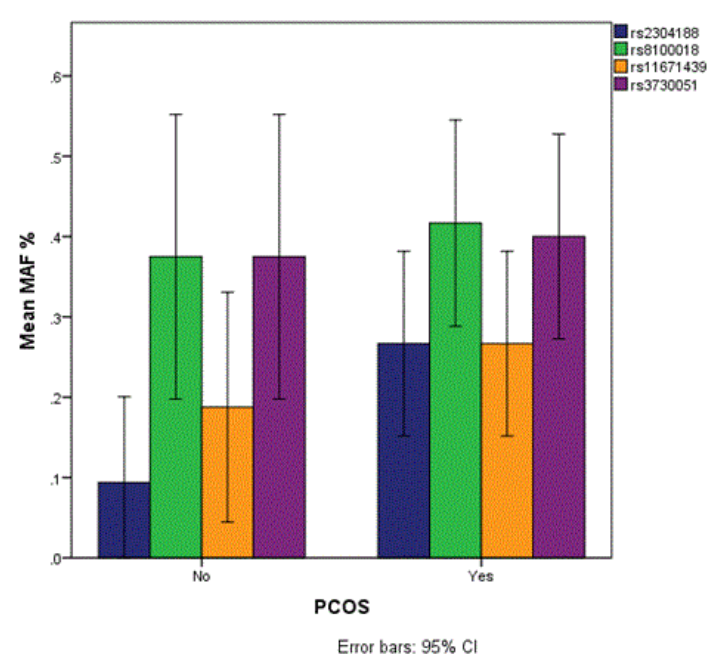

Figure 1: Differences in MAF\% between PCOS cases and controls for all examined $A K T 2$ gene SNPs (error bars denote $95 \%$ CI of each variable percentage presented).

Additionally, there were significantly more cases of hirsutism among those bearing the rs 2304188 polymorphism (92.3\%) than the wild-type carriers (63.4\%, $\mathrm{p}=0.044)$. Regarding the entire population, participants that bear the rs2304188 polymorphism had significantly higher DHEAS (316.8 $\pm 100.1, \quad \mathrm{p}=0.051)$ and higher $17-\mathrm{OH}$ progesterone $(1.02 \pm 0.68, \mathrm{p}=0.009)$. Those with the rs11671439 polymorphism had significantly lower E2 values in the whole population ( $44.4 \pm 13.0, \mathrm{p}=0.040)$ but also when examining the patient group only $(39.1 \pm 10.6, \mathrm{p}=0.025$, Table 5$)$.

\begin{tabular}{|l|l|l|l|}
\hline & Wild-type & Polymorphism & p \\
\hline & Mean (SD) & Mean (SD) & \\
\hline \multicolumn{3}{|l|}{} \\
\hline For the whole population & \multicolumn{2}{l|}{} \\
\hline Differences for rs2304188 & $278.1(126.7)$ & $316.8(100.1)$ & 0.051 \\
\hline $\begin{array}{l}\text { DHEAS } \\
\text { Progesterone }\end{array}$ & $0.64(0.43)$ & $1.02(0.68)$ & 0.009 \\
\hline
\end{tabular}

Differences for rs11671439

\begin{tabular}{|l|l|l|l|}
\hline E2 & $52.5(15.0)$ & $44.4(13.0)$ & 0.040 \\
\hline
\end{tabular}

Only for the patient group

\section{Differences for rs 11671439}

\begin{tabular}{|l|l|l|l}
\hline E2 & $48.8(16.1)$ & $39.1(10.6)$ & 0.025
\end{tabular}

Table 5: Statistically significant differences in serum hormone values for participants bearing various polymorphism of $A K T 2$ gene.

Finally, it was found that eight participants $(13.33 \%$ of cases and $8.7 \%$ of total) had the rs 8100018 polymorphism in combination with either polymorphism rs11671439 or rs2304188. All eight of these participants belonged to the case group ( $\mathrm{PCOS}=100 \%)$. The presence of two polymorphisms was predictive for the presence of PCOS with
$100 \%$ specificity, $13.3 \%$ sensitivity, $100 \%$ positive predictive value and $38.1 \%$ negative predictive value. Of these eight patients, five were having the SNP combination rs8100018-rs11671439 and three were having the SNP combination rs2304188-rs8100018. Specifically, these eight PCOS patients presented with higher $\mathrm{sRANKL}(\mathrm{p}=0.018)$ and 17$\mathrm{OH}$-progesterone $(\mathrm{p}=0.012)$, and lower $\mathrm{E} 2(\mathrm{p}=0.013)$ than all the rest of the entire study group (Figure 2).

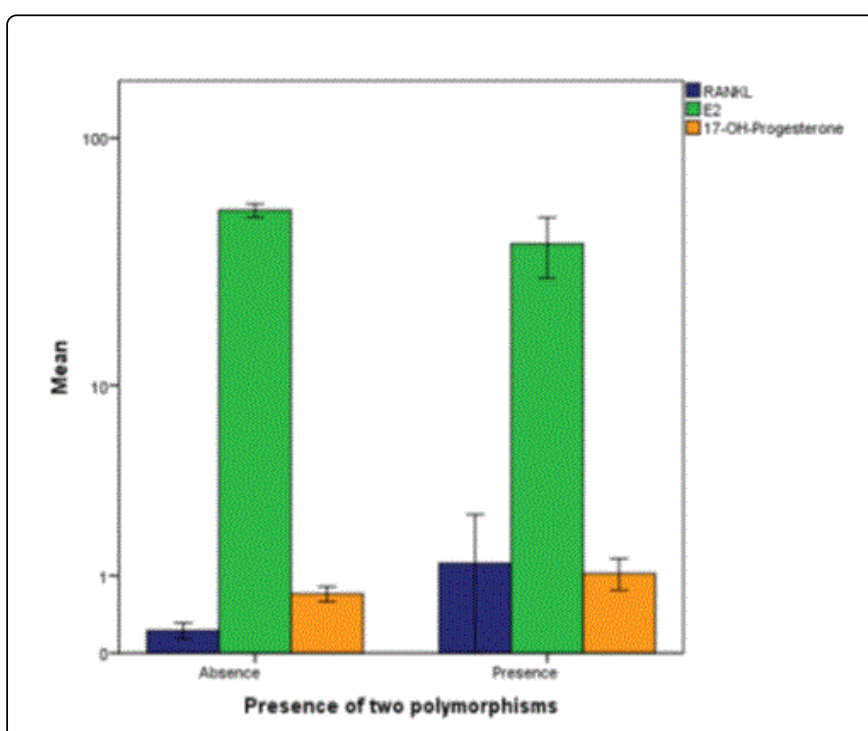

Figure 2: Significant differences within the patient PCOS group regarding serum biochemical markers sRANKL, E2 and 17-OH progesterone in the presence of $A K T 2$ gene rs8100018 in combination with either polymorphism rs11671439 or rs2304188 (error bars denote 95\% CI of the mean value for each variable presented).

\section{Discussion}

Although PCOS is believed to be one of the most common disorders of women, there are few data available regarding its prevalence [18-20]. In the present study, we evaluated the clinical, biochemical and genetic characteristics of women with polycystic ovary syndrome in the Greek population. A strong point of this study is the fact that all ultrasound examinations were performed at one institution, with the same transvaginal ultrasound device and by the same physician who strictly kept to the Rotterdam criteria for diagnosis of PCOS. Our data demonstrate significantly higher serum levels of DHEAS, total testosterone and $17-\mathrm{OH}$ progesterone in the PCOS group compared to the control group, as expected from our well-ascertained patient population. Hyperandrogenemia is the biochemical hallmark of PCOS and women with such a disorder are expected to have elevated levels of DHEAS [21] and testosterone [22-23].

Also, our PCOS group had higher serum levels for sRANKL and significantly lower serum levels for SHBG, E2 and prolactin in comparison to controls. High serum levels of sRANKL have been shown to be a significant predictor of cardiovascular disease [24-25]. Many clinical studies have shown the connection between PCOS and cardiovascular risk due to a lipid/glucose altered metabolism, hypertension, systemic inflammatory condition and vascular injuries [26]. However, there was no significant difference between our two groups regarding OPG values. OPG is a dimeric glycoprotein of the 
TNF receptor family with a molecular weight of $60 \mathrm{kDa}$ and $120 \mathrm{kDa}$ respectively [15]. sRANKL is a member of the tumor necrosis factor (TNF) family, is the main stimulatory factor for the formation of mature osteoclasts and essential for their survival [23]. sRANKL produced by osteoblastic lineage cells and activated T lymphocytes activates its specific receptor RANK that is located on osteoclasts and dendritic cells [23]. OPG acts as a soluble secreted receptor for RANKL and prevents the binding of RANKL to RANK [22,27]. Thus, OPG inhibits RANK activation on the osteoclast surface. The high serum level of sRANKL is a significant predictor of cardiovascular disease $[24,25]$. OPG has a number of biological functions such as antiinflammatory properties and also a protective role of the cardiovascular system. All these functions of OPG are associated with PCOS. Different studies have previously evaluated OPG levels in PCOS patients; however, there is no agreement whether PCOS increases OPG circulating concentration or diminishes it [15]. In concordance with our study, other studies also reported no difference in OPG levels between the two groups [22]. However, there are studies that show either lower OPG levels in PCOS compared to controls [15, 23, 27] or higher OPG levels between the two groups [28]. Although there are many studies that focus on OPG circulating concentration in PCOS, the results vary and this may be due to lack of consensus regarding units, type of sample and antibodies used that target different epitopes in the different ELISA kits used. Since OPG exists both as a monomer and a dimer, its final concentration could differ significantly depending on the kit used.

PCOS seems to run in families. Although genetic factors play an important role in PCOS, the genetic basis of the syndrome remains controversial [29-31]. Our data shows that $A K T 2$ gene polymorphisms could be associated with PCOS or with some of its features. In particular, rs2304188 SNP was found to be more frequent in PCOS patients compared to controls [OR 4.04 (C.I. 1.12-14.54)], whereas concerning the entire population, studied individuals that bear the rs2304188 SNP have higher values of DHEAS and 17-OH progesterone, both biomarkers of PCOS. The rest of the studied SNPs didn't show any correlation in contrast with the only other study -to the best of our knowledge- that has examined $A K T 2$ polymorphisms in PCOS: they showed association with rs3730051 and rs8100018 with OR 2.2 and 2.0 respectively, in a larger US PCOS population [9].

To validate further our $A K T 2$ genetic findings, we compared also our results with those from the GVS for both patients and controls; they showed some differences e.g. for SNP rs3730051 for both patients MAF\% and controls MAF\% and for SNP rs2304188 only for controls MAF\%. However, these differences may be due to the small size of our sample and ethnicity [30]. GVS genotyping data are performed in random populations with no exclusion criteria for PCOS which is a quite common disorder in females. In the present study the examined population was homogenous with regard to racial and ethnic variations.

Also in our study, there were significantly more cases of hirsutism among PCOS patients bearing the rs2304188 polymorphism (92.3\%) that showed association with the disease than those bearing the wildtype $(63.4 \%)$. The most common symptoms of PCOS include hirsutism, irregular menstrual cycles, infertility problems, insulin resistance, dyslipidemia, hypertension, type- 2 diabetes, coronary artery diseases and increased rates of metabolic syndrome [32,33]. Hirsutism is a disorder of excess growth of terminal hairs in androgen-dependent areas in women. The presence or absence of hirsutism depends on whether the increased androgens (produced by both the ovaries and the adrenal glands) are converted peripherally by 5 -alpha reductase to the more potent androgen DHT dihydrotestosterone and 3-alpha diolGlucuronide [34]. Therefore, it is skin 5-alpha reductase activity that determines the presence or absence of hirsutism. Other cutaneous conditions associated with androgen excess are androgenetic alopecia, acanthosis nigricans, and acne $[34,35]$. Hirsutism is often associated with measurably elevated androgen levels, but not in all cases. Androgens in women arise from the ovary and adrenal glands and peripherally from skin and fat. The most common cause of hirsutism is PCOS. Patients with "idiopathic" hirsutism have normal ovulatory cycles and androgen levels [21].

Finally, it was found that eight participants (13.33\% of cases and $8.7 \%$ of total) had the polymorphism of rs8100018 in combination with either rs11671439 or rs 2304188 polymorphism and all of these eight participants were PCOS patients (five were rs8100018 SNPrs11671439 SNP and three were rs2304188 SNP-rs8100018 SNP). However, since a sample of eight people cannot be considered sufficient for drawing reliable conclusions, further studies performing valid haplotype analysis in larger populations are necessary in order to confirm the present results and to firmly establish this genetic association.

Therefore, in our study, rs2304188 SNP shows statistical significant correlation with PCOS and some of its features and AKT2 gene polymorphisms seem worthy of further investigation in larger populations of PCOS. The studied SNPs could be tags for other coding SNP, could affect level of $A K T 2$ mRNA transcript or its splicing; at least 12 different $A K T 2$ splice variants have been detected. The developed $A K T 2$ gene genotyping methods are fast, reproducible and accurate since method comparison with the Gold Standard DNA sequencing has shown $100 \%$ concordance. Also, the aforementioned methods are cost effective and since they use a closed-tube format, they are less prone to contamination.

The genetic etiology of PCOS is still unknown although it is known that a family history of PCOS is common among PCOS patients. This suggests an important genetic component although the mode of inheritance is not yet known. However, since our knowledge of the human genome expands rapidly and new polymorphisms are identified with the advent of novel massive parallel sequencing methods that can easily provide genetic information in panels of multiple genes, this could lead to detailed mapping of all disease loci for PCOS in the near future. This is very important since it will allow family screening and early diagnosis of women with PCOS as well as estimation of the potential risk of developing complications.

\section{References}

1. Fux Otta C, Iraci GS, Szafryk de Mereshian P, Fiol de Cuneo M (2016) Rev Fac Cien Med Univ Nac Cordoba. Polycystic ovary syndrome: impact on reproductive and material fetal health 73: 102-113.

2. Azziz R, Woods KS, Reyna R, Key TJ, Knochenhauer ES, et al. (2004) The prevalence and features of the polycystic ovary syndrome in an unselected population. J Clin Endocrinol Metab 89: 2745-2749.

3. Goodarzi MO, Azziz R (2006) Diagnosis, epidemiology, and genetics of the polycystic ovary syndrome. Best Pract Res Clin Endocrinol Metab 20: 193-205.

4. Escobar-Morreale HF, Luque-Ramirez M, San Millan JL (2005) The molecular-genetic basis of functional hyperandrogenism and the polycystic ovary syndrome. Endocr Rev 26: 251-282.

5. Zawadzki JKDA (1992) Diagnostic criteria for polycystic ovary syndrome: towards a rational approach In: Dunaif AGJ, Haseltine F, 
Citation: Zerva A, Kroupis C, Trakakis E, Poumpouridou N, Tsagkla M, et al. (2017) AKT2 Gene Polymorphisms, Srankl/OPG And Hormone Measurements in Polycystic Ovarian Syndrome (PCOS) Women. Clin Med Biochem 3: 123. doi:10.4172/2471-2663.1000123

Page 7 of 7

editors. Polycystic Ovary Syndromes. Boston: Blackwell Scientific pp. 377-384.

6. Revised 2003 consensus on diagnostic criteria and long-term health risks related to polycystic ovary syndrome (PCOS) (2004) Hum Reprod 19: 41-47.

7. Azziz R, Carmina E, Dewailly D, Kandarakis SE, Escobar-Morreale HF, Futterweit W, et al. (2009) Task Force on the Phenotype of the Polycystic Ovary Syndrome of The Androgen Excess and PCOS Society. The Androgen Excess and PCOS Society criteria for the polycystic ovary syndrome: the complete task force report. Fertil Steril 91: 456-488.

8. Carmina E, Lobo RA (2004) Use of fasting blood to assess the prevalence of insulin resistance in women with polycystic ovary syndrome. Fertil Steril 82: 661-665.

9. Goodarzi MO, Azziz R, Jones MR (2008) First evidence of genetic association between AKT2 and polycystic ovary syndrome. Diabetes Care 31: 2284-2287.

10. Diamanti-Kandarakis E, Papavassiliou A (2006) Molecular mechanisms of insulin resistance in polycystic ovary syndrome. Trends Mol Med 12: 324-332.

11. Vanhaesebroeck B, Alessi DR (2000) The P13K-PDK1 connection: more than just a road to PKB. Biochem J 346: 561-576.

12. Cussons AJ, Stuckey BG, Watts GF (2007) Metabolic syndrome and cardiometabolic risk in PCOS. Curr Diab Rep 7: 66-73.

13. Bozdag G, Mumusuglu S, Zengin D, Karabulut E, Yildiz BO (2016) The prevalence and phenotyping features of polycystic ovary syndrome: a systematic review and meta-analysis. Human Reprod 12: 2841-2855.

14. Zuef LFN, Martins WP, Vieira CS, Ferriani RA (2012) Ultrasonographic and laboratory markers of metabolic and cardiovascular disease in obese women with polycystic ovary syndrome. Ultrasound Obstet Gynecol 39: 341-347.

15. Perez de CC, Varo N (2016) Serum Osteoprotegerin is diminished in the Polycystic Ovary Syndrome and associated with insulin resistance. Biochem Anal Biochem 5: 247.

16. Franks S (2006) Controversy in clinical endocrinology: diagnosis of polycystic ovary syndrome: in defense of the Rotterdam criteria. J Clin Endocrinol Metab 3: 786-789.

17. Azziz R (2003) The evaluation and management of Hirsutism. Obstetrics and Gynecology 101: 995-1007.

18. Zuef LFN, Martins WP, Vieira CS, Ferriani RA (2012) Ultrasonographic and laboratory markers of metabolic and cardiovascular disease in obese women with polycystic ovary syndrome. Ultrasound Obstet Gynecol 39: 341-347.

19. Engmann L, Jin S, Sun F, Legro RS (2017) Racial and ethnic differences in the polycystic ovary syndrome (PCOS). Am J Obstet Gynecol 9378: 30103-30105.

20. Azziz R, Carmina E, Dewailly D (2006) Criteria for defining Polycystic ovary syndrome as a predominantly hyperandrogenic syndrome: An Androgen Excess Society guideline. J Clin Endocrinol Metab 91: 4237-4345.
21. Somani N, Harisson S, Bergfeld WF (2008) The clinical evaluation of hirsutism. Dermatol Ther 5: 376-391.

22. Glintborg D, Hermann AP, Rasmussen LM, Andersen M (2013) Plasma osteoprotegerin is associated with testosterone levels but unaffected by pioglitazone treatment in patients with polycystic ovary syndrome. J Endocrinol Invest 36: 460-465.

23. Pepene CE, Ilie IR, Marian I, Duncea I (2011) Circulating osteoprotegerin and soluble receptor activator of nuclear factor $\mathrm{kB}$ ligand in polycystic ovary syndrome: relationships to insulin resistance and endothelial dysfunction. Eur J Endocrinol 164: 61-68.

24. Sandberg WJ, Yndestad A, Oie E, Ueland T (2006) Enhanced T cell expression of RANK ligand in acute coronary syndrome: possible role in plaque destabilization. Arteriosclerosis, Thrombosis and Vascular Biology 26: 857-863.

25. Kiechl S, Schett JN, Seppi K, Eder P, Egger G, et al. (2007) Soluble receptor activator of nuclear factor-kappa $B$ ligand and risk for cardiovascular disease. Circulation 116: 385-391.

26. Scicchittano P, Dentamaro I, Carbonara R, Bulzis G, Dachille A, et al. (2012) Cardiovascular risk in women with PCOS. Int J Endocrin Metab 10: 611-618.

27. Escobar-Morreale HF, Botella-Carretero JI, Martinez-Garcia MA, LuqueRamirez M, Alvarez-Blasco F, et al. (2008) Serum osteoprotegerin concentrations are decreased in women with the polycystic ovary syndrome. Eur J Endocrinol 159: 225-232.

28. Abali R, Tasdemir N, Alpsoy S, Tasdemir UG, Guzel S, et al. (2015) No relationship between osteoprotegerin concentrations and endothelial dysfunction in non-obese women with and without polycystic ovary syndrome. Arch Gynecol Obstet 291: 1075-1080.

29. Prapas N, Karkanaki A, Prapas I, Kalogiannidis I, Katsikis I, et al. (2009) Genetics of Polycystic Ovary Syndrome. Hippokratia 13: 216-223.

30. Williamson K, Gunn A, Johnson N, Milsom SR (2001) The impact of ethnicity on the presentation of polycystic ovarian syndrome. Aus and New Zeland J of Obst and Gynecology 41: 201-206.

31. Xita N, Georgiou I, Tsatsoulis A (2002) The genetic basis of polycystic ovary syndrome. Europ J Endocr 147: 717-725.

32. Kandarakis DE, Dunaif A (2012) Insulin resistance and the polycystic ovary syndrome revisited: an update on mechanisms and implications. Endocr Rev 33: 981-1030.

33. De Leo V, Musacchio MC, Cappelli V, Massaro MG, Morgante G, et al. (2016) Genetic, hormonal and metabolic aspects of PCOS: an update. Reprod Biol Endocrinol 14: 38.

34. Thigpen AE, Silver RI, Guilleyard JM, Cassey ML, Mc Donell JD, et al. (1993) Tissue distribution and ontogeny of steroid 5-alpha reductase isoenzyme expression. J Clin Invest 92: 903-910.

35. Unluhizarci K, Kaltsas G, Kelestimur F (2011) Non polycystic ovary syndrome-related endocrine disorders associated with hirsutism. Eur J Clin Invest 42: 86-94. 